\title{
Tourette Syndrome Mimic: An Atypical Presentation of Subacute Sclerosing Panencephalitis
}

\author{
Sandeep Paimode ${ }^{1}, M D$ and Jyotindra Narayan Goswami, MD, DNB, DM, MNAMS ${ }^{2 *}$ \\ ${ }^{1}$ Department of Pediatrics, Army Hospital, Amritsar, Punjab, 143001, India \\ ${ }^{2}$ Department of Pediatrics, Army Hospital (R\&R), New Delhi,110010, India
}

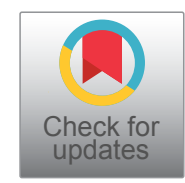

\section{Dear Editor,}

Subacute Sclerosing Panencephalitis (SSPE) is a neurodegenerative disorder caused by measles virus infection [1].We report an atypical presentation of SSPE.

A 10.5-year-old girl presented with abnormal movements and behavioural problems for three months. Initially she used to have lip-twitching and eye-blinking when awake. After six weeks, she started having shoulder-shrugs, neck-turning, throat-clearing at a frequency of 10-20/hour, exacerbated by stress. In addition, she exhibited temper-tantrums, unprovoked crying and scholastic deterioration. She did not have unconsciousness, drop-attacks, limb weakness/tightness, bowel/bladder symptoms, fever, rash, jaundice, head-injury, toxin exposure or contributory family history. Her immunization was complete as per the National Immunization Schedule.

On examination, child was awake, oriented and had normal anthropometry and vital parameters. She had frequent shrugging, teeth-clenching, blinking and tongueclicking which continued despite suggestion / distraction. Her systemic examination, slit-lamp and fundus evaluation were normal. Child was provisionally diagnosed as evolving Tourette syndrome. Her hemogram, liver/renal /thyroid functions, Anti-Thyroid Peroxidase and Anti Nuclear Antibodies, serumceruloplasmin, 24-hour urinary copper, blood lead level, anti-streptolysin $\mathrm{O}$, arterial $\mathrm{pH}$ and lactate were normal. Child's Intelligence Quotient (104) and Pediatric Hurt-InsultThreaten-Scream-Sex (PedHITSS) screen were normal. Her brain Magnetic Resonance Imaging, cerebrospinal fluid (CSF) biochemistry, cytology and autoimmune antibody panel were normal. Electroencephalography revealed frequent, bilateral frontal, 1-2 Hz epileptiform discharges with normal background. She was managed as seronegative autoimmune encephalitis with methyl prednisolone pulse, Intravenous Immunoglobulin and oral clonazepam. Possibility of SSPE was then entertained. Her serum and CSF measles IgG titres and CSF: Serum IgG quotient (EIA) were $8.5 \mathrm{U} / \mathrm{mL}, 84.8 \mathrm{U} / \mathrm{mL}$ and 6.1 (reference normal:1.5) respectively confirming the diagnosis of SSPE. Child was initiated on oral Isoprinosine. At third-month follow-up, she was noted to have myoclonic jerks, cognitive regression and typical EEG.
Subtle intellectual decline followed by progressive myoclonus are initial signature presentations of SSPE which is diagnosed using Dyken's Criteria [1]. Rare atypicalities include dystonia [2], neuropsychiatric manifestations [3] and tics [4]. Motor tics in index case probably served as a precursor of myoclonus. Child's behavioural issues correlated with frontal EEG abnormalities. Atypical periodic discharges as in index case have been reported in SSPE [5].

The case sensitizes readers about an atypical presentation of SSPE mimicking as Tourette syndrome. The symptoms probably occurred as the disease was in an evolving phase.

\section{Authors Contributions}

SP: Patient management, literature review and preparation of draft manuscript.

JNG: Clinician-in-charge of patient management, literature review final approval of manuscript.

\section{Disclosures}

None.

\section{Conflict of Interest}

None.

\section{Funding}

None.

\section{Ethical Clearance}

Not required.

\footnotetext{
*Corresponding author: Jyotindra Narayan Goswami, MD, DNB, DM, MNAMS, Department of Pediatrics, Army Hospital (R\&R), New Delhi:110010, India, Tel: 9893104114

Accepted: July 22, 2021

Published online: July 24, 2021

Citation: Paimode S, Goswami JN (2021) Tourette Syndrome Mimic: An Atypical Presentation of Subacute Sclerosing Panencephalitis. J Pediatr Neurol Neurosci 5(2):138-139
} 


\section{Informed consent}

Obtained from patient's father.

\section{References}

1. Garg RK, Mahadevan A, Malhotra HS, et al. (2019) Subacute sclerosing panencephalitis. Rev Med Virol 29: e2058.

2. Goswami JN, Roy S (2018) Dystonic Storm: An atypical presentation of subacute sclerosing panencephalitis. Indian Pediatr 55: 441.
3. Reddy B, Das S, Guruprasad S (2018) Primary psychiatric manifestations of subacute sclerosing panencephalitis: A case report and literature review. Psychosomatics 59: 408-412.

4. Dey PK, Bhattacharya T (2013) Subacute sclerosing panencephalitis with tics as first symptom. Indian Pediatr 50: 1067-1068.

5. Goswami JN, Sahu JK, Singhi P (2018) An atypical electroencephalographic finding in a child with subacute sclerosing panencephalitis. Journal of Pediatric Neurosciences 13: $284-286$. 